\title{
Facciones cruzadas: acuerdos y disputas partidarias en los cambios de jueces de la Corte de la provincia de Santa Fe, Argentina (1983-2011)
}

\author{
Crossed factions: agreements and partisan disputes in changes of \\ judges at the Court of the province of Santa Fe, Argentina
}

(1983-2011)

\section{Augusto Abdulhadi}

\section{Resumen}

Las Cortes judiciales son órganos colegiados de conducción del Poder Judicial, y tienen poder de decisión sobre temas que afectan proyectos de gobierno o políticas públicas, lo que las convierte en actores relevantes tanto para la política nacional como subnacional. En Argentina existe una rotación frecuente de jueces en los STJ provinciales, y la literatura de estudios judiciales comparados señala que los incentivos partidarios predicen estos cambios en las Cortes. Sin embargo, no existe una clara relación entre estos cambios y el poder partidario de que disponen los gobernadores. Este trabajo realiza un estudio de caso a partir del análisis de los cambios políticos de jueces de la Corte Suprema de la provincia de Santa Fe entre 1983 y 2011. Los mecanismos causales propuestos se basan en un argumento estratégico que sirve para explicar esos cambios políticos de jueces de las Cortes en las provincias argentinas.

\section{Palabras clave}

Política Subnacional; Cortes Judiciales; Democracia; Provincias Argentinas.

\section{Abstract}

Supreme Courts are collegiate organs of conduction of the Judicial Power, and they have decisionmaking power over issues that affect government projects or public policies, which makes them relevant actors for both national and subnational politics. The literature on comparative judicial politics indicates that, in Argentina, Supreme Court judges turnover is frequent, and partisan incentives are a great predictor of changes in Courts, but there is no clear relationship between these changes and the control of legislative majorities. This article presents a case study analysis of the political changes of Supreme Court judges of the province of Santa Fe between 1983 and 2011. The causal mechanisms which are proposed here are based on a strategic argument that explains political changes at Supreme Courts of Argentinean provinces.

\section{Keywords}

Subnational Politics; Supreme Courts; Democracy; Argentine Provinces. 


\section{Introducción ${ }^{1}$}

Este trabajo analiza los cambios políticos de jueces de la Corte Suprema de Justicia (en adelante STJ) de la provincia de Santa Fe, Argentina, en el período 19832011, y propone un argumento estratégico para explicar los cambios de jueces de las Cortes en las provincias argentinas. El argumento se apoya en cuatro mecanismos causales cuyo funcionamiento se explica brevemente en la sección teórica: Imposición, Negociación, Cooptación y Bloqueo.

Los cambios políticos de jueces del STJ de Santa Fe se explican por el mecanismo de Negociación, que implica que el gobernador necesita acordar con otros partidos o facciones partidarias para lograr la mayoría legislativa que le permite efectuar cambios en la Corte. Si bien en el período estudiado se observaron otros mecanismos, no produjeron un cambio político del STJ porque no se alteró el balance de poder partidario al interior de la Corte ${ }^{2}$.

Desde 1983 y hasta 2007 el Partido Justicialista (PJ) reunió más del 50 \% del total de bancas de la Asamblea Legislativa (diputados y senadores provinciales), y por lo tanto dispuso de la mayoría legislativa para designar nuevos jueces en el STJ. Sin embargo, no tuvo en ningún momento por sí solo los dos tercios necesarios para destituir magistrados de la Corte. Esto implica que el gobernador no tenía con su propio partido la mayoría legislativa necesaria para presionar de manera creíble para lograr la renuncia de jueces de la Corte (y así disponer de vacantes para cubrir con nuevos nombramientos). Este trabajo muestra que el respaldo político otorgado por facciones partidarias de oposición permitió al oficialismo concretar los cambios de jueces observados en la Corte provincial. Es por esto que la dinámica de alianzas cruzadas entre partidos y facciones partidarias rivales resulta fundamental para comprender los cambios políticos de jueces del STJ santafesino. El caso de Santa Fe además provee evidencia significativa para el mecanismo del Bloqueo, que implica que un nuevo gobernador presiona para modificar la composición del STJ pero no lo consigue, por ausencia de apoyo político.

\footnotetext{
${ }^{1}$ Este trabajo fue realizado en parte en el marco del proyecto PICT 2014-1712 "Democracia, estado y relaciones estado-sociedad en las provincias argentinas", de la Agencia Nacional de Promoción Científica, Fondo para la Investigación Científica y Tecnológica, dirigido por la Dra. Jacqueline Behrend. El autor agradece los comentarios de las evaluaciones anónimas que permitieron mejorar la versión anterior.

${ }^{2}$ El concepto de cambio político del STJ se explicita y profundiza en la primera sección de este trabajo.
} 
Este artículo se organiza de la siguiente manera. La primera sección presenta el enfoque teórico-metodológico, la propuesta y el argumento. La segunda sección analiza los cambios de jueces del STJ en virtud del mecanismo causal que los explica, el de Negociación, pero también se abordan los demás mecanismos detectados: Bloqueo e Imposición. Los momentos históricos correspondientes están organizados en función del mecanismo causal correspondiente. La tercera sección sintetiza el análisis de los mecanismos que explican los cambios de jueces del STJ en Santa Fe, y finalmente se plantean las conclusiones.

\section{Enfoque teórico, propuesta y argumento}

Las Cortes judiciales son órganos colegiados de conducción del Poder Judicial, y como tienen poder de decisión sobre temas que afectan proyectos de gobierno o políticas públicas, son actores relevantes tanto para la política nacional como subnacional (BILL CHAVEZ, 2004; BRINKS, 2005; HELMKE, 2002; LLANOS et al., 2016; O'DONNELL, 2004; SMULOVITZ, 2010). Y por eso los presidentes y gobernadores buscan ejercer influencia directa sobre las Cortes y expandir lealtades al interior del Poder Judicial (BASABE-SERRANO y POLGA-HECIMOVICH, 2013; CASTAGNOLA, 2012; FINKEL, 2004; INGRAM, 2012; LARA BORGES, CASTAGNOLA y PÉREZ LIÑÁN, 2012).

A diferencia de lo que ocurre en Estados Unidos, donde son los jueces quienes deciden cuándo se retiran de sus cargos (WHITTINGTON, 2007), en América Latina señala es más factible que sea el presidente o los gobernadores, y no los jueces, quienes decidan cuándo se producirá una vacante en las Cortes (CASTAGNOLA, 2012, 2010a; PÉREZ LIÑÁN y CASTAGNOLA, 2009). Entre los hallazgos más relevantes de la literatura de estudios judiciales comparados referida a América Latina, se destaca que un factor clave para predecir nuevos nombramientos en las Cortes tiene que ver con los incentivos partidarios (CASTAGNOLA, 2012, 2010a; LARA BORGES, CASTAGNOLA y PÉREZ-LIÑÁN, 2012; LEIRAS, GIRAUDY y TUÑÓN, 2015; PÉREZ LIÑÁN y CASTAGNOLA, 2009). La llegada de un nuevo gobierno tiene grandes probabilidades de producir cambios en las Cortes ${ }^{3}$.

\footnotetext{
${ }^{3}$ Los únicos casos en América Latina donde se observa una tendencia divergente son Costa Rica, Chile, Uruguay y Brasil, donde las Cortes en el largo plazo muestran mayor estabilidad en su composición, cercana al promedio de la Suprema Corte de Estados Unidos (PÉREZ LIÑ́́N y CASTAGNOLA, 2009).
} 
Otro hallazgo relevante es que, contrariamente a lo esperado desde una visión normativa liberal, las reglas institucionales no dan cuenta de una mayor estabilidad en la composición de las Cortes (LARA BORGES, CASTAGNOLA y PÉREZ-LIÑÁN, 2012). Tampoco hay evidencia que permita sostener que los mandatos vitalicios contribuyen a una mayor estabilidad de los jueces en sus cargos (LARA BORGES, CASTAGNOLA y PÉREZ-LIÑÁN, 2012; LEIRAS, GIRAUDY y TUÑÓN, 2015). Diferentes trabajos coinciden en que las reglas formales del diseño institucional relacionadas con las Cortes no permiten explicar la mayor o menor duración de los jueces en sus cargos (CASTAGNOLA, 2012, 2010a; LARA BORGES, CASTAGNOLA y PÉREZ-LIÑÁN, 2012; LEIRAS, GIRAUDY y TUÑÓN, 2015). Pero la relación entre los incentivos institucionales y la capacidad efectiva de los gobernantes para responder políticamente a esos incentivos es un problema abierto a futuras investigaciones (LARA BORGES, CASTAGNOLA y PÉREZ-LIÑÁN, 2012). Un presidente o gobernador pueden tener fuertes incentivos para presionar a jueces para que renuncien cuando sus partidos controlan la mayoría legislativa necesaria para designar nuevos jueces, pero no cuando otros partidos controlan la mayoría en la legislatura (BILL CHAVEZ, 2004; LARA BORGES, CASTAGNOLA y PÉREZLIÑÁN, 2012). Sin embargo, Lara Borges, Castagnola y Pérez-Liñán (2012) sostienen que en la literatura la cuestión de la interacción de las mayorías legislativas con el diseño institucional es un tema recurrente que requiere mayor profundización histórica.

Uno de los argumentos avanzados por la literatura en relación con el control de las mayorías legislativas afirma que la competencia política favorece la "independencia del poder judicial” (BILL CHAVEZ, 2004; FINKEL, 2004; HELMKE, 2002). Sin embargo, otros trabajos cuestionan este argumento de la competencia política (BASABE-SERRANO y POLGA-HECIMOVICH, 2013; CASTAGNOLA, 2012, 2010a; LEIRAS, GIRAUDY y TUŃÓN, 2015)

En Argentina existe una rotación frecuente de jueces en los STJ provinciales y la literatura de estudios judiciales comparados señala que los incentivos partidarios son un gran predictor de esos cambios en las Cortes (CASTAGNOLA, 2012; LEIRAS, GIRAUDY y TUNÓN, 2015). Sin embargo, no existe una clara relación entre estos cambios y el poder partidario de que disponen los gobernadores (CASTAGNOLA, 2012; LEIRAS, GIRAUDY y TUÑÓN, 2015).

\footnotetext{
4 Basabe-Serrano y Polga-Hecimovich (2013) demuestran el rol que tienen las legislaturas en la inestabilidad judicial, y no sólo el Ejecutivo, pero también señalan, a partir del caso de Ecuador, que la fragmentación del poder político no contribuye a la estabilidad de los jueces de la Corte en sus cargos.
} 
La teoría de los "retiros inducidos" (induced retirements) sostiene que los jueces son inducidos a retirarse a través de estrategias y presiones informales por gobernadores que buscan crear una Corte aliada (CASTAGNOLA, 2012, 2010a). Las estrategias no institucionales, como reconoce Castagnola (2010a), tienen dos desventajas importantes: a) no garantizan el resultado buscado (renuncia de jueces); y b) pueden implicar mucho más tiempo y recursos que una destitución u otra estrategia institucional.

Ahora bien, la teoría de los retiros inducidos (CASTAGNOLA, 2010a) enfoca el problema de manera tal que oscurece el rol de la legislatura y de los líderes partidarios opositores. Para remover jueces de las Cortes eran necesarios dos tercios de bancas legislativas en veintidós (22) de las veinticuatro (24) provincias argentinas en 2009 (CASTAGNOLA, 2010b), con lo cual rara vez un gobernador dispuso con su partido de la mayoría necesaria para remover jueces del STJ. Sin esa mayoría los incentivos partidarios se diluyen porque el gobernador debe negociar con otros actores, ya que las estrategias no institucionales no garantizan las renuncias y vacantes. Pero a pesar de esto los cambios en los STJ fueron frecuentes y la evidencia cuantitativa no muestra una relación clara entre el poder partidario del gobernador y los cambios de jueces del STJ (CASTAGNOLA, 2012; LEIRAS, GIRAUDY y TUŃÓN, 2015).

Los jueces siempre enfrentan presiones y no sólo del titular del Ejecutivo, pero ello no necesariamente lleva a la renuncia de un juez a su cargo en el STJ. Aquí se plantea que el factor decisivo no son las presiones del gobernador, como sostiene Castagnola (2010a), sino el respaldo político que da credibilidad a esas presiones (para desplegar represalias).

El concepto de "cambio político de jueces del STJ" implica que hay cambios de jueces que alteran el balance de poder partidario al interior de la Corte. Los indicadores utilizados son dos: a) el cambio en la pertenencia partidaria de la mayoría de jueces del STJ; o b) cuando no cambia la pertenencia partidaria de la mayoría que prevalece en la Corte, pero cambia más del $50 \%$ de la composición del STJ. Este segundo indicador permite capturar casos en los que hay una reestructuración profunda del STJ por disputas entre facciones partidarias, aunque gobierne el mismo partido.

Por facciones partidarias se entiende a los grupos internos de los partidos que, sobre la base del control de ciertos recursos, compiten por espacios de poder y candidaturas del partido (LEIRAS, 2007), y cuyos miembros comparten un sentido de identidad y actúan en forma colectiva para alcanzar sus objetivos (BOUCEK, 2009). 
Desde este enfoque teórico, este trabajo propone cuatro mecanismos causales para explicar los cambios políticos de jueces del STJ en las provincias argentinas: 1) Imposición; 2) Negociación; 3) Cooptación; y 4) Bloqueo. Los tres primeros explican los cambios de jueces en los STJ de acuerdo con el contexto político (Cuadro 1). El Bloqueo explica los intentos fallidos de cambio político de jueces de la Corte.

El mecanismo de Imposición implica que el partido del gobernador dispone por sí solo de la mayoría necesaria para designar jueces en la Corte y no necesita negociar con otros actores (siempre que la mayoría de su partido efectivamente responda a su liderazgo) $)^{5}$.

Cuadro 1 - Mecanismos causales y cambios de jueces del STJ

\begin{tabular}{|l|c|c|}
\hline $\begin{array}{l}\text { Mecanismo } \\
\text { Causal }\end{array}$ & Contexto político & Resultado STJ \\
\hline Imposición & Gobierno mayoritario & $\begin{array}{c}\text { El gobernador designa a los jueces que prefiere } \\
\text { en el STJ sin necesidad de negociar }\end{array}$ \\
\hline Negociación & $\begin{array}{c}\text { Gobierno minoritario } \\
\text { con acuerdo partidario }\end{array}$ & $\begin{array}{c}\text { Se modifica la composición del STJ por } \\
\text { negociación y acuerdo entre diferentes partidos o } \\
\text { facciones partidarias }\end{array}$ \\
\hline Cooptación & $\begin{array}{c}\text { Poder partidario del } \\
\text { gobernador cercano a umbral } \\
\text { de mayoría necesaria }\end{array}$ & $\begin{array}{c}\text { Se modifica la composición del STJ por } \\
\text { cooptación de legisladores individuales que } \\
\text { respaldan la iniciativa del gobernador }\end{array}$ \\
\hline Bloqueo & $\begin{array}{c}\text { Gobierno minoritario sin } \\
\text { acuerdo partidario }\end{array}$ & $\begin{array}{c}\text { A pesar de las presiones del gobernador, no } \\
\text { hay cambio en STJ }\end{array}$ \\
\hline
\end{tabular}

Fuente: Elaboración propia.

El mecanismo de Negociación implica que el partido del gobernador negocia y acuerda con otros partidos o facciones para modificar la Corte. La negociación puede ser entre diferentes partidos o entre facciones partidarias de diferentes partidos.

El mecanismo de Cooptación se diferencia sustancialmente del de Negociación, porque el partido del gobernador reúne un porcentaje de bancas legislativas muy cercano a la mayoría necesaria y con cooptar el voto de algunos legisladores individuales es suficiente para cambiar jueces de la Corte.

Por último, el mecanismo del Bloqueo explica los intentos fallidos de cambio político del STJ por parte de gobernadores que, aunque tienen la intención de

\footnotetext{
${ }^{5}$ El gobernador no siempre era el jefe partidario, y la mayoría partidaria no respondía necesariamente a sus iniciativas.
} 
modificar la Corte y presionan a los jueces para forzar su salida, no consiguen cambiar la composición del STJ.

Lo que diferencia los mecanismos de Negociación, Cooptación y Bloqueo es la agencia de los actores. La acción de los actores involucrados cambia el resultado.

\section{Metodologia}

La pregunta que orienta este trabajo se aborda con un estudio de caso, que es el método más apropiado para investigar mecanismos y realizar inferencias causales (MAHONEY y GOERTZ, 2006). Se utilizó este método porque se busca desarrollar una teoría y refinar hipótesis teóricas sobre los mecanismos causales (FALLETI y LYNCH, 2009) que explican los cambios políticos de jueces de la Corte en diferentes contextos.

Santa Fe constituye un caso de interés para investigar y explorar mecanismos causales (GOERTZ, 2013) porque los cambios de jueces de la Corte ocurrieron sin que mediara alternancia partidaria, una variable clave para la literatura (CASTAGNOLA, 2012; LEIRAS, GIRAUDY y TUÑÓN, 2015). Y cuando hubo alternancia, y el nuevo gobernador cuestionó al STJ, no hubo cambios.

Los estudios de caso abordan, por definición, casos positivos. Pero la provincia de Santa Fe también aporta evidencia negativa relevante. El "principio de posibilidad" (MAHONEY y GOERTZ, 2004) permite distinguir la evidencia negativa relevante de la irrelevante. La evidencia negativa relevante surge por el hecho de que, contra la expectativa teórica (CASTAGNOLA, 2012) hubo momentos de alternancia partidaria, con un gobernador que presionó públicamente por la renuncia de algunos jueces de la Corte y sin embargo estos no renunciaron. El fenómeno de interés podía ocurrir, por alternancia partidaria y por presiones del gobernador, pero no ocurrió, y este trabajo sostiene que esto se explica por el mecanismo del Bloqueo.

Este estudio de caso es parte de un trabajo más amplio que está basado en las siguientes técnicas de recolección de datos:

a) Base de datos original con información sobre el porcentaje de bancas legislativas del partido del gobernador, y los momentos de alternancia partidaria en cada provincia argentina entre 1983 y el año 2011 (datos del Atlas Electoral de Andy Tow [2011]). Esta base de datos contiene, para cada provincia argentina, el porcentaje de la bancada legislativa que tenía el partido o coalición del gobernador al momento de su elección, y las variaciones de ese porcentaje en elecciones intermedias - legislativas. Santa Fe no tiene elecciones legislativas intermedias, así que el dato coincide con la elección de 
gobernador. La base de datos permite, además, identificar cuándo hubo alternancia partidaria, cuándo hubo alternancia ejecutiva y cuándo fue reelecto un gobernador;

b) Base de datos original con información sobre la totalidad de jueces que integraron las Cortes provinciales de las provincias argentinas (Santa Fe en este caso), con las fechas de ingreso y egreso del cargo de cada juez. Esta base contiene apellido y nombre de la persona que integró el STJ, fecha de ingreso al cargo, fecha de egreso (o si sigue en el cargo), y una columna de observaciones, para el período estudiado (19832011);

c) Base de datos propia con información de fuentes secundarias sobre la evolución de los procedimientos de designación y remoción, así como sobre las mayorías necesarias para designar y remover jueces de los STJ en las provincias argentinas. En este caso de Santa Fe;

d) Trabajo de hemeroteca. Se revisaron los Diarios de Sesiones de la Asamblea Legislativa de Santa Fe y los principales diarios de la prensa escrita local de la provincia de Santa Fe, con el fin de identificar las principales facciones partidarias en cada caso, y con el propósito de analizar los momentos de cambio político de jueces de los STJ. Las fechas de ingreso y egreso de jueces de las Cortes orientaron este trabajo de archivo.

e) Entrevistas con informantes claves. Las entrevistas se realizaron a informantes claves para el acceso a información no disponible por otros medios (RATHBUN, 2010). En especial se hicieron con actores protagonistas del proceso político en torno a los cambios de jueces de la Corte santafesina. El trabajo de hemeroteca y las entrevistas son complementarias y se utilizaron para triangular la información de las bases de datos para el caso de Santa Fe, ya que no se trata de una muestra estadística. El trabajo de hemeroteca y las entrevistas sirvieron también para identificar presiones de los gobernadores para cambiar la composición de las Cortes. Al final del artículo, después de las referencias bibliográficas, se incluye un anexo que resume las entrevistas realizadas. El trabajo se complementó con fuentes secundarias: informes oficiales y bibliografía existente.

Aunque el referente empírico es un caso provincial (Santa Fe) y ello impone cautela al plantear conclusiones, los mecanismos propuestos están pensados para analizar los cambios de jueces de las Cortes más allá del caso (SNYDER, 2001). De esta manera se busca promover la reflexión teórica sobre casos comparables, tanto de otras provincias argentinas como de otros países federales (BEHREND y WHITEHEAD, 2016) donde los estados o provincias tienen Cortes judiciales con niveles de autonomía similar. 


\section{Cambios de jueces en el STJ santafesino por mecanismo causal}

Esta sección presenta el análisis del caso de Santa Fe organizado en torno a los mecanismos causales detectados: Negociación, Imposición y Bloqueo. El mecanismo de Negociación explica los cambios políticos de jueces de la Corte provincial, y el de Bloqueo explica por qué los gobernadores no consiguieron el cambio político de la Corte a pesar de haberlo intentado. El mecanismo de Imposición sólo se detectó en una coyuntura particular pero no produjo un cambio político del STJ porque el peronismo (PJ) ya tenía mayoría en el STJ. Los diferentes momentos históricos están organizados en función del mecanismo causal correspondiente. En primer lugar, se presenta el mecanismo de Negociación, luego el de Bloqueo y por último el de Imposición.

\section{El mecanismo de Negociación}

Los momentos de cambio político en el STJ santafesino se explican de acuerdo con el mecanismo de Negociación. En este apartado se reseñan los momentos de cambio en el STJ, divididos en los dos períodos destacados en el Cuadro 2. La primera parte está dedicada a los cambios de jueces en el período 1983-1991 y los acuerdos cruzados entre el PJ y el Partido Radical (UCR), y la segunda parte está centrada en los cambios realizados durante el período 1999-2003, donde también se verifican acuerdos cruzados entre PJ y UCR.

Cuadro 2 - Cambios de jueces del STJ Santa Fe 1983-2011

\begin{tabular}{|c|c|}
\hline Período & Cambios en STJ \\
\hline & Mayo y junio 1988 cambian dos jueces \\
& (reemplazados inmediatamente) \\
& Diciembre 1989 (renuncia un juez) \\
& Mayo 1990 (se nombra) un juez \\
& Junio 1991 (renuncia) un juez \\
& Noviembre 1991 (se nombra) un juez \\
\hline \multirow{2}{*}{$1999-2003$} & Febrero 2000 (renuncia un juez) \\
& Mayo 2000 (renuncia un juez) \\
& Junio 2000 (se nombran dos jueces) \\
& Septiembre 2000 (renuncia un juez) \\
& Septiembre 2001 (renuncia un juez) \\
& Octubre 2001 (se nombran dos jueces) \\
\hline
\end{tabular}

Fuente: Elaboración propia.

Nota: entre noviembre de 1991 y hasta el año 2000 no hubo ningún cambio de juez en el STJ santafesino. 


\section{Disputas y acuerdos PJ-UCR después del retorno a la democracia (1983-1991)}

En 1983 el peronismo ganó la gobernación de Santa Fe con José María Vernet, un candidato respaldado por la Unión Obrera Metalúrgica (UOM) y los sindicatos, que predominaban dentro del partido (MAINA, 2013). El peronismo santafesino tenía mayoría en la Asamblea Legislativa, pero el partido estaba muy fragmentado por disputas internas, que incluso derivaron en un juicio político y la destitución de un vicegobernador en 1990 (LASCURAIN, 2015). El PJ en las Cámaras de Senadores y Diputados provinciales se dividía en dos bloques (MAINA, 2013), ninguna de las facciones en disputa lograba imponerse sobre sus rivales, y los legisladores peronistas no necesariamente respondían todos al gobernador (entrevista con Ángel Baltuzzi, 24/05/2018; entrevista con 1SF, 14/05/2018) ${ }^{6}$. El STJ provincial tuvo cambios de jueces en poco tiempo en este período, al ritmo de las disputas entre facciones partidarias del PJ y de la UCR.

El gobernador Vernet (PJ) promovió la designación de los seis nuevos jueces del STJ en diciembre de 1983. Los dirigentes de "la cooperativa" del $\mathrm{PJ}^{7}$ administraban las designaciones de Vernet (entrevista con Roberto Rosúa, 24/05/2018), incluyendo la de los jueces de la Corte. Y de los seis jueces designados en 1983, cuatro tenían vínculos con el PJ, uno con la UCR, y uno era más cercano a los democratacristianos (entrevista con 1SF, 14/05/2018).

El siguiente gobernador del PJ fue Víctor Reviglio (1987-1991). Durante su gobierno los conflictos internos y las disputas entre facciones del peronismo se acentuaron e impactaron en la composición del STJ. Entre mayo de 1988 y noviembre de 1991 cambiaron cuatro de los seis jueces del STJ (Tabla 1).

El triunfo de un candidato outsider de la política, con apoyo del peronismo nacional, modificó el mapa de poder local del PJ santafesino (entrevista con Ángel Baltuzzi, 24/05/2018) ${ }^{8}$. En el STJ existía una vacante que no fue cubierta por casi cinco meses, y el saliente gobernador Reviglio, tras el triunfo de Reutemann (outsider), se propuso cubrirla antes de dejar el cargo. En la Asamblea Legislativa se hicieron

\footnotetext{
${ }^{6}$ Las disputas al interior de la UCR no eran menos intensas que en el PJ en los años 1980 (MAINA, 2013).

7 "La Cooperativa” se denominó al conjunto de sectores que dirigía el PJ en los 1980 (LASCURAIN, 2015).

${ }^{8}$ Reutemann era un outsider de la política en ese momento, pero tuvo respaldo del presidente Menem y del PJ nacional para ser el candidato local del peronismo (LASCURAIN, 2015).
} 
evidentes las diferencias al interior del PJ, aunque la mayoría de los legisladores peronistas votaron a favor del candidato propuesto por Reviglio?. Un senador del PJ cuestionó al gobernador saliente y sostuvo lo siguiente:

[...] algunos compañeros legisladores que no están presentes en esta sesión, porque quieren expresar de esta manera su disconformidad por la metodología y la oportunidad de la remisión de los pliegos [...] quería venir aquí a decirles, a mis compañeros, que voy a votar negativamente [...] Creo que el gobierno que se está yendo [...] no tiene legitimidad política para seguir generando remisión de pliegos y nombramientos. (SANTA FE, 1991, p. 14).

También votaron a favor seis legisladores de una facción de la UCR y un diputado de la "Unidad Socialista" que se separó de su bloque. El nuevo juez del STJ propuesto por el gobernador saliente (Reviglio), identificado como un político peronista (SANTA FE, 1991), recibió el apoyo del $68 \%$ del total de la Asamblea. Con esa designación de noviembre de 1991, la composición del STJ santafesino se mantuvo sin cambios hasta el año 2000.

\section{Apoyo cruzado: el acuerdo entre Reutemann (PJ) y Usandizaga (UCR) para los cambios politicos del STJ santafesino entre 2000 y 2001}

A fines de los años 1990 Reutemann había consolidado su liderazgo al interior del PJ santafesino (LASCURAIN, 2015; RAMOS, 2011). Ante las elecciones de 1999 los realineamientos internos favorecieron a la facción reutemista, que retuvo la estructura partidaria y recuperó el control de la mayoría del PJ en la Cámara de Diputados ${ }^{10}$. Así inició Reutemann su segundo mandato como gobernador (19992003) y jefe del PJ.

Apenas inició su segunda gestión comenzó el proyecto de reestructurar el STJ. Entre febrero y mayo del 2000, el gobernador consiguió la renuncia de dos jueces del STJ provincial, uno de ellos de origen radical, otro vinculado con el PJ (entrevista con

\footnotetext{
${ }^{9}$ Los diputados y senadores de Santa Fe se renuevan completamente cada cuatro años. Por ello la Asamblea Legislativa estaba compuesta por legisladores electos en 1987, cuando Reviglio accedió a la gobernación.

${ }^{10}$ En el Senado provincial la facción reutemista tuvo control de la mayoría del PJ desde el inicio de la gestión de Obeid en 1995 (RAMOS, 2011).
} 
1SF, 14/05/2018). El primero de los jueces renunciantes se retiró apenas cumplió la edad en la que la Constitución de la provincia de Santa Fe (CPSF) dispone que pierden la inamovilidad, pero el segundo juez, a pesar de su cercanía con el PJ, resistió los intentos del gobernador Reutemann. "El Dr. Barraguirre, tengo que decir, cuando cumplió 65 años vino y puso la renuncia a disposición del gobierno. Se fue por su voluntad. Los otros tres, hubo arduas negociaciones, que las hice yo personalmente" (entrevista con Ángel Baltuzzi, 24/05/2018).

La renuncia del segundo juez se dio en el contexto de presiones del ministro de gobierno de Reutemann y un subsecretario. Para evitarle un juicio político al juez de la Corte, el peronismo santafesino le exigió la renuncia (SANSO DE LA MADRID, 2000a; entrevista con Ángel Baltuzzi, 24/05/2018).

Estas dos vacantes fueron cubiertas en junio del 2000, cuando la Asamblea Legislativa aprobó los pliegos de dos candidatos remitidos por el gobernador (SANTA FE, 2000). El PJ no contaba por sí solo con los dos tercios de la Asamblea Legislativa para remover a un juez. Pero el acuerdo con el líder de la facción radical "Convergencia" (Horacio Usandizaga) permitió al gobernador Reutemann tener el respaldo del 80 \% del total de la Asamblea Legislativa ${ }^{11}$. Votaron a favor 40 diputados y senadores del PJ y 15 legisladores de la UCR enrolados en la facción "Convergencia”. Las críticas realizadas por algunos legisladores opositores estaban centradas en los ostensibles vínculos partidarios y personales de los nuevos jueces con el gobernador (LA ASAMBLEA..., 2000) ${ }^{12}$.

El acuerdo con el líder radical de "Convergencia" le garantizó al gobernador la mayoría legislativa que daba credibilidad a las presiones informales para que renuncien los jueces del STJ. Así logró Reutemann nombrar los primeros dos jueces de la Corte vinculados con su liderazgo.

Poco después continuó la ofensiva del gobernador por lograr nuevas vacantes, y en septiembre de 2000 renunció al STJ otro juez que, en este caso, ya había conseguido un aval de la Corte Suprema nacional para permanecer en el cargo.

"Pero en algún momento que había habido un intento de mover a dos
miembros que eran los más grandes, Iribarren y Álvarez, se fueron con un
amparo a la Corte nacional, y la Corte nacional que también tenía interés

\footnotetext{
${ }^{11}$ Es decir 55 de los 66 legisladores presentes (SANTA FE, 2000).

${ }^{12}$ Uno por ser pariente del gobernador y el otro por haber sido su abogado personal y representante del Poder Ejecutivo en el Consejo de la Magistratura.
} 
propio en el tema, le hizo lugar a una cautelar por lo cual permanecieron en sus cargos." (Entrevista con Ángel Baltuzzi, 24/05/2018).

Casi un año después, a fines de agosto de 2001, el gobernador consiguió finalmente la renuncia de otro juez del STJ, que ya a fines del 2000 había anunciado su intención de retirarse (RAÚL..., 2000). Este alejamiento era esperado por el gobernador, justificado en que este juez excedía los 65 años de edad previstos en la CPSF a partir de lo cual pierden la inamovilidad ${ }^{13}$. Desde comienzos del ańo $2001 \mathrm{el}$ ministro de gobierno de Reutemann sostenía que esperaban el alejamiento de este juez para promover los nuevos nombramientos y cubrir dos vacantes al mismo tiempo en la Asamblea Legislativa (DUCLÓS, 2001).

Así, en septiembre de 2001 la Asamblea Legislativa aprobó los dos nombramientos del STJ enviados por el gobernador. Si bien la Corte ya tenía mayoría de jueces designados por el peronismo, se trató de un cambio político de jueces del STJ como se definió en la sección primera, ya que implicó una reestructuración de más del $50 \%$ de la Corte: se renovaron cuatro jueces de un total de seis. El análisis de los nombramientos, a partir del trabajo de hemeroteca y de los Diarios de Sesiones de la Asamblea Legislativa, permitió advertir el acuerdo político entre el gobernador Reutemann y el líder de la facción radical "Convergencia”, y las presiones informales desplegadas sobre jueces que finalmente renunciaron a sus cargos. Sin embargo, no sólo se trató de presiones informales sino de un bloque legislativo que disponía de la amenaza de destitución. Las últimas dos personas nombradas en el STJ por Reutemann fueron una mujer con carrera política (previamente diputada provincial por el PJ) y un juez camarista del Poder Judicial de Santa Fe y amigo del líder de la facción radical "Convergencia", Horacio Usandizaga (MARIO..., 2001). Así se completó la reestructuración de la Corte santafesina promovida por el gobernador Reutemann con apoyo de una facción radical.

\footnotetext{
${ }^{13}$ El artículo 88 de la CPSF sostiene que a los 65 los jueces pierden la inamovilidad en el cargo. Un fallo dictado por la Corte Suprema de Justicia de la Nación el 22/6/1999 favoreció al juez del STJ santafesino que se encontraba alcanzado por ese artículo constitucional. De todos modos, renunció en septiembre de 2000 .
} 


\section{E1 mecanismo del Bloqueo: fragmentación legislativa y estabilidad del STJ}

En este apartado se presentan los casos en que los gobernadores tuvieron intención de cambiar el STJ pero no lo consiguieron. El argumento de por qué no pudieron concretar los cambios que buscaban es porque no reunieron el apoyo legislativo necesario. El mecanismo del Bloqueo se detectó en el primer mandato de Reutemann (1991-1995) y en el mandato de Binner (2007-2011) luego de la alternancia partidaria en 2007.

\section{E1 rechazo del propio partido: Reutemann en 1995}

En los inicios del gobierno de Reutemann, no todo el PJ local respondía a sus iniciativas (LASCURAIN, 2015). De hecho, fueron varias las ocasiones en que Reutemann no consiguió que la legislatura apruebe sus iniciativas. Para el momento en que Reutemann consolidó su liderazgo dentro del PJ santafesino (LASCURAIN, 2015), ya se encontraba en los últimos tramos de su primer mandato y debía dejar el cargo porque la CPSF no permitía la reelección inmediata del gobernador.

En lo que respecta a los nombramientos del STJ, propuso un candidato propio como procurador general de la Corte. Sin embargo, en la sesión del 10 de junio 1995 la Asamblea Legislativa rechazó los pliegos del candidato del gobernador (NUEVA..., 1995). En ese tiempo la disputa más importante dentro del PJ era entre los dirigentes del "norte" provincial (con epicentro en la capital) y los del "sur" (con centro en Rosario $)^{14}$. El control partidario del PJ por parte de la facción que respondía a Reutemann se incrementó durante el transcurso de la primera gestión de Obeid (19951999), específicamente luego de las elecciones de 1997 (RAMOS, 2011), y el sector que respondía a Obeid cedió paso a la hegemonía reutemista, que se fortaleció progresivamente en los ańos siguientes ${ }^{15}$.

Una explicación rival del rechazo al candidato del gobernador Reutemann podría ser que el candidato promovido no reuniera los antecedentes necesarios. Sin embargo, el mismo candidato fue propuesto por Reutemann en 2000 para ser juez del STJ, y en esa ocasión el nombramiento sí prosperó. Por lo tanto, las disputas entre

\footnotetext{
${ }^{14}$ El clivaje territorial entre el norte y el sur santafesino excede al PJ y abarca a todos los actores. Respecto a los opositores internos a Reutemann, se nucleaban sobre todo en el sur provincial (LASCURAIN, 2015).

${ }^{15}$ El fortalecimiento de su facción interna le permitió al gobernador Reutemann, al asumir su segundo mandato, acordar con la facción radical de "Convergencia" y realizar cambios políticos de jueces del STJ.
} 
facciones partidarias rivales y la falta de negociación de un acuerdo político, activaron el mecanismo del Bloqueo, que implicó el rechazo a la iniciativa del gobernador.

\section{Alternancia partidaria, fragmentación legislativa y el Bloqueo 2007-2011}

El PJ contó sistemáticamente con mayoría propia, tanto en la Cámara de Diputados como en la de Senadores provinciales, al menos hasta 2007. Ese año el "Frente Progresista Cívico y Social" (FPCyS) ganó las elecciones y obtuvo mayoría en la Cámara de Diputados de la provincia. Sin embargo, el PJ consiguió mantener su mayoría en la Cámara de Senadores. Por ello el partido del gobernador Binner no logró reunir ni siquiera el $50 \%$ de la Asamblea Legislativa.

Una de las promesas de campaña de Hermes Binner fue que en caso de ganar las elecciones promovería en Santa Fe un cambio en el STJ similar al que desplegó Néstor Kirchner como presidente de la Nación respecto de la Corte Suprema de Justicia nacional ${ }^{16}$. Si bien Binner no llegó a comprometerse públicamente a promover un juicio político a los integrantes de la Corte santafesina, sí cuestionó en duros términos a los jueces designados en virtud del acuerdo Reutemann-Usandizaga (TERMINAR..., 2006), y especialmente los nombramientos en el STJ. Sin embargo, a pesar de sus cuestionamientos, la composición de la Corte no sufrió cambios durante la gestión del partido socialista (PS).

El FPCyS no reunía la amenaza de destitución, y las presiones del gobernador sobre los miembros de la Corte provincial no fueron efectivas para forzar la renuncia de jueces de la Corte. Un legislador socialista que además fue funcionario de la administración, graficó la situación del siguiente modo:

"Se hicieron los cambios posibles en el Poder Judicial, no los necesarios; [...] Pero lo que no se hizo, fue por falta de poder, no de voluntad política: llevamos en Santa Fe tres gobiernos consecutivos en la más absoluta minoría en el Senado, y con una mayoría endeble en la Cámara de Diputados. Es más: el gobierno de Bonfatti tuvo las dos Cámaras Legislativas en manos

\footnotetext{
${ }^{16}$ Néstor Kirchner, a poco de asumir como presidente de la nación, modificó procedimientos para designar jueces de la Corte Suprema de la Nación. En paralelo, también promovió el juicio político a los jueces de la Corte Suprema nacional más notoriamente vinculados con el menemismo, en un contexto de amplio descrédito hacia la denominada "mayoría automática menemista" (BRINKS, 2005).
} 
del peronismo [...] En ese marco, claramente se hace lo que se puede [...]." (Entrevista con Alfredo Luis Cecchi, 24/04/2018).

Por ello, a pesar de las críticas formuladas por el gobernador Binner, incluso las fuertes presiones desplegadas en ocasión del fallo sobre el caso "Fraticelli" ${ }^{17}$, los jueces del STJ no renunciaron a sus cargos. Y esto se explica porque los jueces del STJ contaban con respaldo político de partidos y facciones partidarias que podían sostenerlos en sus cargos ${ }^{18}$. La clave no son las presiones del gobernador sobre los jueces por medio de estrategias no institucionales, sino el respaldo político del que gozan los jueces del STJ. Específicamente, importa si existen partidos o facciones partidarias que puedan bloquear eventuales represalias del partido del gobernador. "Eso también hace que para el gusto de algunos y sin entrar en detalles, el poder judicial sigue siendo un enclave peronista, pero nunca se tuvo, ni remotamente, la posibilidad de hacer lo que se consideraba necesario hacer" (entrevista con Alfredo Luis Cecchi, 24/04/2018).

No obstante lo anterior, Binner sí realizó cambios importantes en sintonía con los realizados a nivel nacional por el presidente Kirchner: un decreto de autolimitación sobre el procedimiento para seleccionar a futuros jueces del STJ (BINNER..., 2007), y la jerarquización del Consejo de la Magistratura provincial.

Una explicación rival sobre por qué el gobernador Binner no cambió a los jueces del STJ heredados del período del peronismo (ni pidió juicio político), podría ser porque en su visión un gobernador no debe influir en la Corte. Sin embargo, justamente esa razón (la creencia en la neutralidad del STJ respecto al gobernador) sería un argumento para intentar cambiar la composición de la Corte provincial con jueces más neutrales, ampliamente aceptados por las diversas fuerzas políticas de la provincia. Por ese motivo se argumenta aquí que Binner no pudo cambiar la composición del STJ santafesino dada la ausencia de respaldo legislativo, y sus cuestionamientos públicos a los jueces del STJ revelan su intención de que renuncien.

\footnotetext{
${ }_{17}$ Un fallo de la Corte Suprema de la Nación (CSJN) absolvió a una pareja que había sido acusada y condenada por la muerte de su hija adolescente. Pero el fallo de la CSJN realizó duros cuestionamientos al sistema penal de la provincia de Santa Fe por no garantizar la imparcialidad del proceso. En ese contexto, al conocerse el fallo de la CSJN, el gobernador Binner criticó en duros términos a la Corte santafesina, y sostuvo que sus integrantes debían renunciar.

${ }^{18}$ De hecho, ex funcionarios del gobierno de Reutemann entre 1999-2003 respondieron públicamente a las críticas de Binner desafiando al gobernador a pedir juicio político y presentar las pruebas que correspondan (FUERTES..., 2009), sabiendo que el oficialismo no reunía el respaldo legislativo necesario.
} 


\section{El mecanismo de Imposición}

En 2003 el candidato del peronismo, Jorge Obeid, fue electo gobernador para un nuevo período (2003-2007). En 2004 se realizó una reforma política que eliminó la ley de lemas y estableció las internas abiertas, simultáneas y obligatorias como paso previo a la elección general (RAMOS, 2011). Con estas nuevas reglas electorales se concretó la alternancia partidaria y el candidato del Partido Socialista, Hermes Binner, fue electo gobernador para el período 2007-2011 por la coalición del Frente Progresista Cívico y Social.

Si bien ya había sido electo un nuevo gobernador (Binner), poco tiempo antes de dejar el cargo el todavía gobernador Jorge Obeid envió varios pliegos para nombrar jueces a la legislatura provincial. En lo que interesa a este trabajo, una semana antes de las elecciones provinciales de septiembre de 2007, renunció a su cargo en el STJ un juez que llevaba diecinueve años en la Corte (RODOLFO..., 2007). La vacante liberada en la Corte fue objeto de polémica a raíz de las denuncias y acciones de un legislador del socialismo santafesino que promovió una investigación que desembocó en la anulación del beneficio jubilatorio (ANULARON..., 2009).

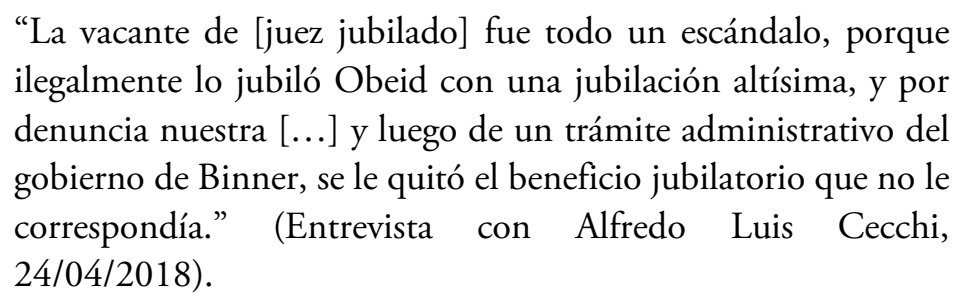

Con el triunfo de Binner consumado, el gobernador Obeid decidió de todos modos enviar a la Asamblea Legislativa el pliego de un nuevo candidato a integrar la Corte provincial. El candidato promovido por Obeid para cubrir la vacante en el STJ había sido afiliado al partido radical, y fue votado no sólo por legisladores del peronismo, sino que también fue respaldado por el voto de legisladores de la UCR, aunque no hubo un acuerdo político con el radicalismo sino que se trató de una decisión unilateral del gobernador Obeid ${ }^{19}$. En la votación se abstuvieron un diputado del PJ, y con duras críticas al gobernador Obeid y al procedimiento de selección de

\footnotetext{
${ }^{19}$ El entonces ministro de gobierno de Obeid afirmó que se trató de una cuestión netamente personal del gobernador, sin que mediara un acuerdo expreso con la UCR (entrevista con Roberto Rosúa, 24/05/2018).
} 
jueces del STJ, se abstuvieron también legisladores del PS, el partido Alternativa por una República de Iguales (ARI) y el Partido Demócrata Progresista (PDP).

Obeid envió no sólo el pliego del candidato a juez, sino también el de un candidato para ocupar el cargo de Procurador General de la Corte. Ambos pliegos fueron tratados en conjunto, pero la votación fue separada en cada caso. Esta particularidad permitió ver que más allá del acompañamiento de los legisladores de la UCR para el juez del STJ propuesto por Obeid, el peronismo de todos modos contaba con los votos legislativos necesarios para imponer la designación. De hecho, el candidato a Procurador General sólo cosechó los votos de los legisladores del PJ (37 votos a favor) y la oposición de la UCR, el PS y el ARI (23 votos negativos). Uno de los legisladores del PS, entre los más críticos de la actitud asumida por el gobernador Obeid, juzgaba que el verdadero objetivo era asegurar a un procurador general leal al peronismo, y tenía margen para ser generoso con el radicalismo:

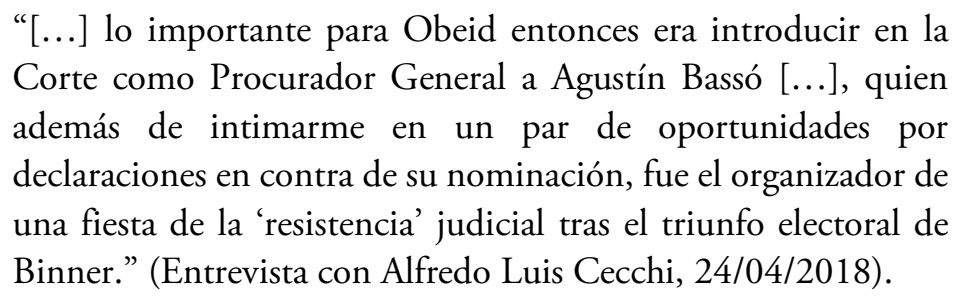

Fueran cuales fueran los motivos del gobernador Obeid, y más allá del apoyo legislativo de algunos legisladores radicales, el mecanismo fue de Imposición en la medida en que no hubo negociación ni cooptación.

\section{Acuerdos cruzados entre facciones partidarias, mecanismos causales y cambios en el STJ santafesino}

Los cambios de jueces de la Corte en el caso de Santa Fe durante el período analizado (1983-2011) se explican por el mecanismo de Negociación. Las disputas internas, tanto en el PJ como en la UCR, se encuentran detrás de aquellos acuerdos que permiten explicar los cambios políticos de jueces en el STJ santafesino.

Cuando Reutemann accedió a su segundo mandato como gobernador (19992003) impulsó cambios en la Corte provincial, y logró designar a cuatro nuevos jueces entre junio del 2000 y octubre de 2001 (SANTA FE, 2000, 2001). Pero lo hizo a partir de un acuerdo con una facción partidaria de la UCR (SANSO DE LA MADRID, 2000b). El peronismo reunía en la Asamblea Legislativa la mayoría necesaria para 
designar un nuevo juez en el STJ, pero no contaba con los dos tercios necesarios para impulsar un juicio político a los jueces que se encontraban en uso del cargo. Las estrategias no institucionales con las cuales el gobernador Reutemann logró presionar y obtener la renuncia de cuatro jueces de la Corte estaban respaldadas por más de dos tercios de la Asamblea Legislativa, a partir del acuerdo con el líder de la facción radical mayoritaria, Horacio Usandizaga. Eso tornaba creíbles las presiones del gobernador (entrevista con Ángel Baltuzzi, 24/05/2018).

De hecho, los cuatro jueces del STJ designados por Reutemann entre $2000 \mathrm{y}$ 2001 consiguieron más de dos tercios de votos del total de la Asamblea Legislativa, porque fueron respaldados por el peronismo y por los radicales del sector de "Convergencia" (SANTA FE, 2000, 2001). El acuerdo con la facción de "Convergencia" era necesario para reunir la amenaza de destitución y presionar de manera efectiva la renuncia de jueces del STJ. Sin la mayoría efectiva para destituir en caso de negativa, las negociaciones informales no hubieran garantizado el resultado, o bien hubieran requerido mucho más tiempo (entrevista con Ángel Baltuzzi, 24/5/2018).

Por otro lado, antes de dejar su cargo de gobernador en 1991, Reviglio (PJ) promovió la designación de un juez para completar la composición del STJ. La iniciativa contó con el respaldo de buena parte del peronismo ("la Cooperativa"), pero también de un sector de la UCR (SANTA FE, 1991).

La llegada de Reutemann a la gobernación en 1991 modificó el mapa de poder en el PJ local. Durante el primer mandato de Reutemann (1991-1995) y de Obeid (1995-1999) no se observaron presiones sistemáticas e intentos de cambiar la composición de la Corte. Aun así, las disputas entre sectores rivales dentro y entre los partidos generaron escenarios de fragmentación, incluso en el partido mayoritario. Por ello, cuando Reutemann intentó designar como procurador general de la Corte a un abogado cercano, su propio partido rechazó los pliegos (NUEVA..., 1995). El sector del PJ liderado por Reutemann sólo logró convertirse en hegemónico a nivel provincial a fines de los años 1990 (LASCURAIN, 2015).

Uno de los aportes de este trabajo es poner en cuestión el supuesto frecuente en la literatura de política subnacional según el cual se asume que los gobernadores son los jefes partidarios de sus respectivas fuerzas políticas. Esto no es necesariamente así y por tanto la mayoría legislativa no necesariamente responde a sus iniciativas. Durante los gobiernos de Jorge Obeid (1995-1999 y 2003-2007), el jefe partidario del peronismo provincial fue Reutemann (RAMOS, 2011). Pero a su vez Reutemann, que 
llegó a la gobernación en 1991 como outsider, no era el jefe partidario, sino que fue construyendo su liderazgo en el PJ santafesino a partir de disputar poder dentro del partido (LASCURAIN, 2015) ${ }^{20}$.

En el Cuadro 3 se especifica el porcentaje de bancas del partido o coalición del gobernador para cada período legislativo considerado en este trabajo. Se tomó la Asamblea Legislativa provincial porque el interés está en la mayoría requerida por el diseño institucional para designar y para remover jueces del STJ.

Cuadro 3 - Gobernadores y porcentaje de escaños de su partido en la Asamblea Legislativa. Santa Fe (1983-2011)

\begin{tabular}{|l|c|c|c|}
\hline Período & Gobernador & Partido & $\begin{array}{c}\text { Bancas legislativas } \\
\text { partido del } \\
\text { gobernador (en \%) }\end{array}$ \\
\hline $1983-1987$ & Vernet & PJ & 53,62 \\
\hline $1987-1991$ & Reviglio & PJ & 63,76 \\
\hline $1991-1995$ & Reutemann & PJ & 62,31 \\
\hline $1995-1999$ & Obeid & PJ & 59,42 \\
\hline $1999-2003$ & Reutemann & PJ & 62,31 \\
\hline $2003-2007$ & Obeid & PJ & 62,31 \\
\hline $2007-2011$ & Binner & PS/FPCyS & 47,82 \\
\hline
\end{tabular}

Fuente: Elaboración propia sobre datos del Atlas Electoral de Andy Tow (2011).

El Cuadro 3 muestra que todos los gobernadores peronistas dispusieron de la mayoría legislativa necesaria para designar jueces en el STJ (hasta 2007). Con la alternancia partidaria de 2007 el PJ pierde esa mayoría. Sin embargo, para remover jueces de la Corte se necesita un juicio político que exige dos tercios de cada Cámara (Diputados y Senadores provinciales). Ningún gobernador reunió esa mayoría con su partido por sí solo, por lo cual era necesario un acuerdo político con otros partidos o facciones partidarias para alcanzar la amenaza de destitución.

El argumento de Castagnola $(2012,2010 \mathrm{a})$ es que el gobernador presiona a los jueces para obtener vacantes en la Corte y designar aliados suyos. Y esas presiones se realizan a través de estrategias no institucionales, que son menos costosas y menos visibles desde el punto de vista del gobernador. Esto se observa efectivamente en el segundo mandato de Reutemann en Santa Fe con los cambios que tuvieron lugar

\footnotetext{
${ }^{20}$ En 1995 el gobernador Reutemann propuso un candidato para procurador general de la Corte que fue rechazado hasta por los propios legisladores del PJ (NUEVA..., 1995). Pero la misma persona fue propuesta por Reutemann en 2000 y esta vez sí la Asamblea Legislativa respaldó el nombramiento.
} 
entre 2000 y 2001. Pero el argumento de Castagnola (2012, 2010a) deja afuera de la explicación por qué esas presiones del gobernador por medios no institucionales son efectivas. Ése es precisamente el aporte de este trabajo, al mostrar que las presiones o amenazas del gobernador sólo son creíbles para los jueces si el gobernador dispone de la mayoría legislativa necesaria para removerlos en caso de que los jueces no accedan a retirarse (sea mayoría propia, o una mayoría lograda a través del mecanismo de Negociación o el de Cooptación). De lo contrario, esas presiones o amenazas caen en el vacío, como le ocurrió al gobernador socialista Binner (2007-2011) ${ }^{21}$.

\section{Conclusiones}

En primer lugar, el caso de Santa Fe demuestra que resulta fundamental profundizar el análisis más allá de la composición de los bloques partidarios en el ámbito legislativo. El poder partidario del gobernador nos informa sobre la fuerza relativa de cada bloque partidario, pero la dinámica política no necesariamente traduce ese poder en respaldo constante a las iniciativas del gobernador. Aun cuando el partido del gobernador dispone de la mayoría necesaria para designar nuevos jueces del STJ, no puede darse por sentado que la mayoría responda al gobernador. Incluir los acuerdos, negociaciones y pactos entre los líderes de partidos y facciones partidarias en el ámbito legislativo en futuros trabajos, permitiría considerar una variable clave del proceso político.

En segundo lugar, el caso de Santa Fe nos informa también sobre el rol de las oposiciones partidarias, un tema soslayado en la literatura de estudios judiciales comparados $^{22}$ donde el foco está puesto en los presidentes o gobernadores como los responsables de las reestructuraciones de las Cortes judiciales. El caso de Santa Fe permite ver que el rol de partidos o facciones partidarias de oposición al gobernador es clave en la medida que pueden bloquear o facilitar cambios en los STJ.

Por último, cuando un gobernador quiere cambiar la Corte provincial pero no tiene suficiente respaldo político en el ámbito legislativo (poder encargado de la

\footnotetext{
${ }^{21}$ Otros casos de Bloqueo similares detectados en otras provincias argentinas son los siguientes: a) Chaco entre 2007-2011, donde el nuevo gobernador presiona públicamente a jueces del STJ, que no renuncian; b) Ciudad de Buenos Aires entre 2007-2011: el nombramiento promovido por el Jefe de Gobierno fue bloqueado en la legislatura; c) Misiones entre 2003-2005, donde los jueces del STJ tenían respaldo de UCR y PJ; d) Chaco en 1993, cuando el PJ logró bloquear juicio político contra el STJ promovido por la UCR y aliados de Acción Chaqueña (ABDULHADI, 2020).

${ }^{22}$ Una excepción la constituye el trabajo de Basabe-Serrano y Polga-Hecimovich (2013).
} 
remoción de jueces del STJ), la acción de pedir públicamente sus renuncias parece ser una estrategia no institucional como las que sugiere Castagnola (2010a), aunque el caso de Binner en Santa Fe no produjo ese resultado. Los jueces no renuncian necesariamente ante presiones del gobernador, sino que la clave está en el respaldo político de que disponen por parte de partidos o facciones partidarias que pueden sostenerlos en sus cargos.

Augusto Abdulhadi é Doutor em Ciência Política e Membro da Área Política e Subnacional e Relações Intergovernamentais da Escola de Politica e Governo da Universidad Nacional de San Martín (EPYG-UNSAM). E-mail: aabdulhadi@unsam.edu.ar ou augustoabdulhadi@gmail.com.

\section{Referências}

ABDULHADI, Augusto. Cortes y democracia en Argentina: una propuesta teórica. Latin American Law Review, n. 4, p. 73-106, 2020. doi: 10.29263/lar04.2020.04

ANULARON la jubilación del ex ministro Rodolfo Vigo. El Litoral, 28 dic. 2009. Disponible en: $<$ https:/www.ellitoral.com/index.php/id_um/48096-anularon-la-jubilacion-del-ex-ministro-rodolfovigo>. Acceso: 18 ago. 2021.

BASABE-SERRANO, Santiago; POLGA-HECIMOVICH, John. Legislative Coalitions and Judicial Turnover under Political Uncertainty: The Case of Ecuador. Political Research Quarterly, v. 66, n. 1, p. 154-166, 2013.

BEHREND, Jacqueline; WHITEHEAD, Laurence. Illiberal Practices. Territorial Variance within Large Federal Democracies. Johns Hopkins University Press, 2016.

BILL CHAVEZ, Rebecca. The Rule of Law in Nascent Democracies: Judicial Politics in Argentina. Stanford, CA: Stanford University Press, 2004.

BINNER firmó el decreto de autolimitación. El Litoral, 13 dic. 2007. Disponible en: <https://www.ellitoral.com/index.php/id_um/26805->. Acceso: 18 ago. 2021. 
BOUCEK, Francoise. Rethinking Factionalism. Typologies, Intra-Party Dynamics and Three Faces of Factionalism. Party Politics, v. 15, n. 4, p. 1-31, 2009. doi: 10.1177/1354068809334553

BRINKS, Daniel. Judicial Reform and Independence in Brazil and Argentina: the beginning of a new millennium? Texas International Law Journal, v. 40, p. 595-622, 2005.

CASTAGNOLA, Andrea. I want it all, and I want it now: the political manipulation of Argentina's Provincial High Courts. Journal of Politics in Latin America, v. 4, n. 2, p. 39-62, 2012. doi: $10.1177 / 1866802 X 1200400202$

CASTAGNOLA, Andrea. Rethinking Judicial Instability in Developing Democracies: A National and Subnational Analysis of Supreme Courts in Argentina. 2010. 232 p. Tesis (Doctorado en Philosophy in Political Science) - Graduate Faculty of Arts and Sciences, University of Pittsburgh, Pittsburgh, 2010a.

CASTAGNOLA, Andrea. La diversidad institucional de los poderes judiciales provinciales en Argentina desde una perspectiva histórica. POST-Data, v. 15, n. 2, p. 161-189, $2010 \mathrm{~b}$.

DUCLÓS, Carlos. Esperan la renuncia de Alvarez para modificar la Corte. La Capital, 20 feb. 2001. Disponible en: <https://archivo.lacapital.com.ar/2001/02/20/articulo_158.html>. Acceso: 18 ago. 2021.

FALLETI, Tulia G.; LYNCH, Julia. Context and Causation in Political Analysis. Comparative Political Studies, v. 49, n. 9, p. 1143-1166, 2009.

FINKEL, Jodi. Judicial Reform in Argentina in the 1990s: How electoral incentive shape institutional change. Latin American Research Review, v. 39, n. 3, p. 56-80, 2004.

FUERTES reacciones por las críticas de Binner a la Corte. El Litoral, 24 nov. 2009. Disponible en: <https://www.ellitoral.com/index.php/id_um/47094-fuertes-reacciones-por-las-criticas-de-binner-ala-corte?utm_source=relacionadas $>$. Acceso: 18 ago. 2021.

GOERTZ, Gary. Case studies, causal mechanisms, and selecting cases. Kroc Institute for International Peace Studies University of Notre Dame, 2013. Part I.

HELMKE, Gretchen. The logic of Strategic Defection: Court-Executive Relations in Argentina under Dictatorship and Democracy. American Political Science Review, v. 96, n. 2, p. 291-303, 2002.

INGRAM, Matthew. Crafting Courts in New Democracies: Ideology and Judicial Council Reforms in Three Mexican States. Comparative Politics, v. 44, n. 4, p. 439-458, 2012.

LA ASAMBLEA Legislativa les dará luz verde a los pliegos. La Capital, 08 jun. 2000.

LARA BORGES, Oswald; CASTAGNOLA, Andrea; PÉREZ LIÑÁN, Aníbal. Diseño constitucional y estabilidad judicial en América Latina, 1900-2009. Política y Gobierno, v. 19, n. 1, p. 87-114, 2012.

LASCURAIN, María Cecilia. El peronismo santafesino en el poder: crisis y recomposición de la representación política a partir de la constitución del liderazgo de Carlos Reutemann (1989-1995). 2015. 203 p. Tesis (Maestría en Ciencia Política) - Instituto de Altos Estudios Sociales, Universidad Nacional de General San Martin, Buenos Aires, 2015.

LEIRAS, Marcelo. Todos los caballos del rey. La integración de los partidos políticos y el gobierno democrático de la Argentina, 1995-2003. Buenos Aires: Prometeo-PENT, 2007.

LEIRAS, Marcelo; GIRAUDY, Agustina; TUÑÓN, Guadalupe. Who wants an independent court? Political competition and supreme court autonomy in the Argentine provinces (1984-2008). Journal of Politics, v. 77, n. 1, p. 175-187, 2015. 
LLANOS, Mariana et al. Informal interference in the judiciary in new democracies: a comparison of six African and Latin American cases. Democratization, v. 23, n. 7, p. 1236-1253, 2016. doi: 10.1080/13510347.2015.1081170

MAHONEY, James; GOERTZ, Gary. A Tale of Two Cultures: Contrasting Quantitative and Qualitative Research. Political Analysis, n. 14, p. 227-249, 2006. doi: 10.1093/pan/mpj017

MAHONEY, James; GOERTZ, Gary. The Possibility Principle: Choosing Negative Cases in Comparative Research. The American Political Science Review, v. 98, n. 4, p. 653-669, 2004.

MAINA, Marcelino. Oficialismo y oposición durante la transición a la democracia en la provincia de Santa Fe. In: JORNADAS INTERESCUELAS/DEPARTAMENTOS DE HISTORIA, 14., 2013, Mendoza. Actas de la XIV Jornada Interescuelas/Departamentos de Historia. Mendoza: UNCUYO, Facultad de Filosofía y Letras, 2013.

MARIO Netri: "llego a la Corte por antecedentes, no por ser amigo de Usandizaga". La Capital, 07 sep. 2001. Disponible en: <https://archivo.lacapital.com.ar/2001/09/07/articulo_45.html>. Acceso: 18 ago. 2021.

NUEVA derrota del gobernador. La Capital, p. 1-2, 02 jun. 1995.

O'DONNELL, Guillermo. The Quality of Democracy: Why the Rule of Law Matters. Journal of Democracy, v. 15, n. 4, p. 32-46, 2004.

PÉREZ LIÑÁN, Aníbal; CASTAGNOLA, Andrea. Presidential Control of High Courts in Latin America: a long-term view (1904-2006). Journal of Politics in Latin America, v. 1, n. 2, p. 87-114, 2009. doi: 10.1177/1866802X0900100204

RAMOS, Hugo. Metamorfosis y crisis de representación. Las estrategias electorales del PJ santafesino en las elecciones provinciales de 1999 y 2003. Revista SAAP, v. 5, n. 1, p. 157-187, 2011.

RATHBUN, Brian C. Interviewing and Qualitative Field Methods: Pragmatism and Practicalities. In: BOX-STEFFENSMEIER, Janet M.; BRADY, Henry E.; COLLIER, David (Eds.). The Oxford Handbook of Political Methodology. Oxford, UK: Oxford University Press, 2010.

RAÚL Alvarez dejará su cargo en la Corte Suprema de Justicia. La Capital, 28 nov. 2000. Disponible en: <https://archivo.lacapital.com.ar/2000/11/28/articulo_177.html>. Acceso: 18 ago. 2021.

RODOLFO Vigo se va de la Corte Suprema. El Litoral, 22 ago. 2007. Disponible en: <https://www.ellitoral.com/index.php/id_um/24077->. Acceso: 18 ago. 2021.

SANSO DE LA MADRID, Jorge. Decio ulla se fue de la Corte. La Capital, p. 19, 18 mayo 2000a.

SANSO DE LA MADRID, Jorge. Juraron los nuevos jueces de la Suprema Corte santafesina. La Capital, p. 21, 22 jun. 2000b.

SANTA FE. Diario de Sesiones de la Asamblea Legislativa, 04 de octubre de 2001. Santa Fe, 2001.

SANTA FE. Diario de Sesiones de la Asamblea Legislativa, o8 de junio de 2000. Santa Fe, 2000.

SANTA FE. Diario de Sesiones de la Asamblea Legislativa, 31 de octubre de 1991. Santa Fe, 1991.

SMULOVITZ, Catalina. Judicialization in Argentina: Legal Culture or Opportunities and Support Structures? In: COUSO, Javier; SIEDER, Rachel; HUNEEUS, Alexandra (Eds.). Cultures of Legality. Judicialization and Political Activism in Latin America. Cambridge University Press, 2010.

SNYDER, Richard. Scaling down: The Subnational Comparative Method. Studies in Comparative International Development, v. 36, n. 1, p. 93-110, 2001. 
210 | Augusto Abdulhadi

TERMINAR con la impunidad. Rosario12, 23 abr. 2006. Disponible en: <https:/www.pagina12.com.ar/diario/suplementos/rosario/10-3180-2006-04-22.html>. Acceso: 18 ago. 2021.

TOW, Andy. Atlas Electoral. 2011. Blog de Andy Tow. Disponible en: <https://www.andytow.com/blog/>. Acceso: 5 abr. 2018.

WHITTINGTON, Keith E. Presidents, Senates, and Failed Supreme Court Nominations. The Supreme Court Review, p. 401-438, 2007.

Texto recebido em 26 de janeiro de 2021. Aprovado em 15 de julho de 2021. 


\section{Anexo I - Entrevistas realizadas a informantes clave de la provincia de Santa Fe}

\begin{tabular}{|c|c|c|}
\hline Nombre/Código & Antecedentes & Fecha de entrevista \\
\hline $\begin{array}{c}\text { Alfredo Luis } \\
\text { Cecchi }\end{array}$ & $\begin{array}{c}\text { Diputado provincial del PS entre 1991-2007 y } \\
\text { funcionario y dirigente socialista }\end{array}$ & $24-04-2018$ \\
\hline Ángel Baltuzzi & $\begin{array}{c}\text { Ex ministro de Gobierno de Reutemann. Ex } \\
\text { senador y dirigente del PJ }\end{array}$ & $24-05-2018$ \\
\hline Juan Lewis & $\begin{array}{c}\text { Ex ministro de Justicia y Derechos Humanos del } \\
\text { PS, y ex subsecretario del ministro de Justicia y } \\
\text { Derechos Humanos de Binner }\end{array}$ & $25-05-2018$ \\
\hline Roberto Rosúa & $\begin{array}{c}\text { Ex ministro de Gobierno de Obeid, y ex dirigente } \\
\text { del PJ }\end{array}$ & $24-05-2018$ \\
\hline 1SF & $\begin{array}{c}\text { Ex dirigente del PJ. Ex funcionario de los } \\
\text { gobiernos de José María Vernet y Víctor Reviglio }\end{array}$ & $14-05-2018$ \\
\hline 2SF & Ex periodista del diario La Capital de Rosario & $15-05-2018$ \\
\hline
\end{tabular}

